Martin Laun, Dietmar Auhl, Rüdiger Brummer, Dirk J. Dijkstra, Claus Gabriel, Marc A. Mangnus, Maximilian Rüllmann, Wim Zoetelief and Ulrich A. Handge*

\title{
Guidelines for checking performance and verifying accuracy of rotational rheometers: viscosity measurements in steady and oscillatory shear (IUPAC Technical Report)
}

\begin{abstract}
The paper addresses techniques for checking the performance of rotational rheometers with coneplate, plate-plate, or concentric cylinder geometry. We focus on the determination of the viscosity as a function of the shear rate and of the magnitude of the complex viscosity as a function of the angular frequency. After summarizing the relevant definitions and test modes, we show examples of measurements in the linear viscoelastic range, and applications of the Cox-Merz relationship. Sources of reference fluids with defined viscosities are presented, and their use in tests for verification of accuracy is demonstrated. Relevant issues, predominantly for Newtonian reference liquids, are the exploration of measurement limits, related either to the shear rate range or to reliably accessible viscosity levels. Viscoelastic reference samples are also discussed. Prerequisites for sample preparation and loading are addressed. In particular, we present recommendations based on experience from various laboratories. Finally, we discuss the problem of temperature calibration, presenting techniques that allow the determination of the true sample temperature for a given set temperature of the rheometer. This paper summarizes contributions from various industrial and academic laboratories.
\end{abstract}

Keywords: IUPAC Polymer Division; macromolecular chemistry; materials chemistry; polymers; rheology; viscoelasticity.

DOI 10.1515/pac-2013-0601

Received June 3, 2013; accepted June 25, 2014

Article note: Sponsoring body: IUPAC Polymer Division; see more details on p. 1966.

\footnotetext{
*Corresponding author: Ulrich A. Handge, Institute of Polymer Research, Helmholtz-Zentrum Geesthacht, Max-Planck-Strasse 1, 21502 Geesthacht, Germany, e-mail: ulrich.handge@hzg.de

Martin Laun: (Retired Scientist), 71638 Ludwigsburg, Germany

Dietmar Auhl: Faculty of Humanities and Sciences, Maastricht University, MD 6200 Maastricht, The Netherlands

Rüdiger Brummer: (Retired Scientist), Rüdiger Brummer, 21255 Tostedt, Germany

Dirk J. Dijkstra: Bayer MaterialScience AG, Physics/Rheology, 51368 Leverkusen, Germany

Claus Gabriel and Maximilian Rüllmann: BASF SE, 67056 Ludwigshafen, Germany

Marc A. Mangnus: Dow Benelux B.V., Plastics R\&D, Terneuzen NL-4530, The Netherlands

Wim Zoetelief: DSM Research, PM-MSC, NL-6167 MD Geleen, The Netherlands
} 


\section{CONTENTS}

1. INTRODUCTION

2. RELATED LITERATURE.............................................................................................................1947

3. MEASUREMENT MODES AND REGIMES OF OPERATION............................................................1947

3.1 Constant shear rate measurement ..................................................................................1948

3.2 Shear creep test.............................................................................................................1949

3.3 Oscillatory shear measurement ...............................................................................1950

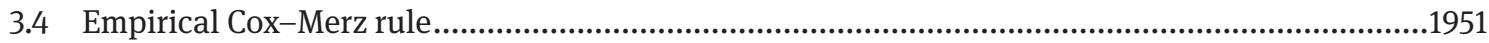

3.5 Comparison of widely used tool geometries.................................................................. 1952

4. REFERENCE MATERIALS ................................................................................................ 1954

4.1 Sources of viscosity reference materials......................................................................... 1954

4.2 Newtonian and non-Newtonian calibration liquids.......................................................... 1954

5. EXPLORATIONS OF RHEOMETER MEASUREMENT LIMITS......................................................1955

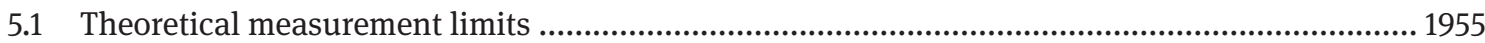

5.2 Practical determination of reliable measurement ranges....................................................... 1957

6. ROUTINE CALIBRATION PROCEDURES........................................................................................1962

6.1 Performance check: Calibration and verification ................................................................ 1962

6.2 Effect of actual sample temperature on the viscosity ...........................................................1963

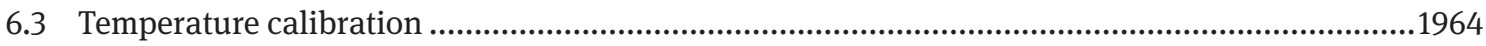

7. SUMMARY .........................................................................................................1965

8. MEMBERSHIP OF SPONSORING BODY .......................................................................... 1966

ACKNOWLEDGMENTS ........................................................................................................ 1966

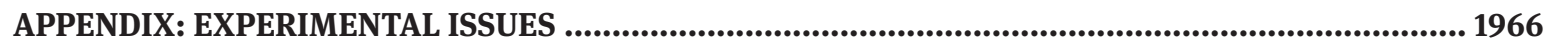

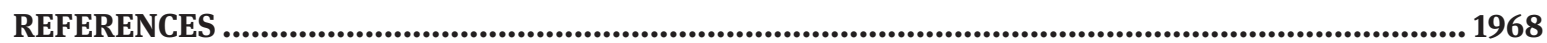

\section{Introduction}

After decades of technical improvements, modern commercial rheometers are user-friendly instruments: They provide a state-of-the-art mechanical setup (low-friction bearings, efficient temperature control, sensitive torque, and angular deflection sensors), automated measurement protocols, and user-friendly software to analyse the measured data. Consequently, the various instruments should yield comparable data sets.

In reality, this goal is not always achieved for every type of sample, as repeatedly demonstrated by interlaboratory comparisons. For example, a recent IUPAC round-robin test of polyolefin melts (at present unpublished) revealed differences of approximately $40 \%$ in the reported zero-shear rate viscosity $\eta_{0}$ ! All laboratories involved in the study claimed a high level of experience in rheological testing. This disappointing result shows that generating reliable rheology data still remains a challenge.

Rheometers of today have a much higher torque resolution than those available 30 years ago, yet there remain measurement limits. Additional problems include the determination of the true sample temperature and its uniformity within the shear gap, the resolution of the angular deflection measurement, and the stiffness of the rheometer (which affects gap opening caused by normal force and angular deflection caused by torque). Instrument-related limitations are complemented by sample-related challenges even for thermally stable materials: the occurrence of secondary flows in viscoelastic melts may cause erroneous viscosity data, and dissipative heating results in a reduction of viscosity with time. Knowledge about these effects and their magnitude for the sample under investigation helps to avoid misinterpreting the readings.

The objective of this paper is to provide guidelines for checking the performance and limitations of shear rheometers. We focus on standard applications like the determination of the shear viscosity function (shear viscosity $\eta$ versus shear rate $\dot{\gamma}$ in steady shear flow) as well as on the determination of the magnitude of the complex viscosity $\left|\eta^{*}\right|$ as a function of the angular frequency $\omega$ or the time $t$, using small amplitude oscillatory 
shear. Various industrial and academic rheology laboratories collaborated to merge their expertise. The present paper thus goes beyond the content of ordinary manuals and is meant to help and guide rheometer users.

\section{Related literature}

Macosko has published a comprehensive textbook about the principles of rheometry [1]. Mainly practical issues of rheometry are treated in refs. [2-4]. The rheological and processing properties of polymer melts are addressed in refs. [5-15]. Marquardt and Nijman discuss the accuracy of rotational rheometers and possible sources of error [16]. Geometrical errors (e.g., tool dimensions out of tolerance) are treated and also the origin of erroneous torque and deflection signals.

A publication of the National Institute of Standards and Technology (NIST) addresses the certification of rheological properties of a viscoelastic reference material [17]. It also covers sources of uncertainty and error propagation for viscosity functions and dynamic moduli measurements, like the gap error arising from the presence of air when zeroing the gap in plate-plate (PP) geometry [18]. Gabriel and Kaschta [19] discuss the performance of stress-controlled rotational rheometers in measurement modes such as creep and creep recovery. Velankar and Giles [20] present a procedure for checking the accuracy of phase angle measurements close to $\delta=\pi / 2$ in oscillatory shear, which is relevant to materials of low elasticity.

The precision of viscosity measurements using oscillatory shear at either imposed stress or strain amplitude is compared in ref. [21]. Typically imposed stress amplitude gives more accurate data at low frequencies. On the other hand, imposed strain amplitude experiments are more appropriate in the high-frequency regime. Consequently, controlled strain rheometers should use the "variable strain technique," implying an automatic increase in the strain amplitude below a certain frequency or stress amplitude. However, there is the risk of applying strain amplitudes beyond the linear viscoelastic range (see Section 5.2).

Barnes and Bell [22] address the historical development of stress-controlled rheometers, starting with the first devices driven by weights and pulleys around 1910 and ending with state-of-the-art equipment. These instruments allow creep tests to be performed even at very low shear stresses.

\section{Measurement modes and regimes of operation}

This paper is restricted to rotational rheometry, at either imposed shear rate or imposed shear stress or strain. The imposed stress and strain, respectively, may be kept constant with time or oscillate sinusoidally. These two modes cover the majority of standard rheological tests. We start by briefly defining the relevant rheological parameters. More details can be found in textbooks, e.g., ref. [1]. For simplicity, we begin with cone-plate geometry, which has the advantage of providing an approximately uniform shear rate and shear stress across the whole gap for small cone angles. Experimental examples are used to show the response of a typical material and also to define the regime of linear viscoelasticity. Other test geometries like PP or concentric cylinders are addressed later.

In the cone-plate (CP) torsional rheometer, the sample is sheared in the gap between a lower, horizontal plate of radius $R$ and a truncated cone of angle $\alpha$ with the identical radius $R$. An angular displacement (angle $\varphi$ ) between cone and plate causes a shear strain $\gamma$ which is given for small cone angles by [1]

$$
\gamma=\frac{\varphi}{\tan \alpha}
$$

The uniform shear strain yields a uniform shear stress $\tau$ in the sample, which is measured via the resulting torque $M$ 


$$
\tau=\frac{3 M}{2 \pi R^{3}}
$$

\subsection{Constant shear rate measurement}

Imposing an angular velocity $\dot{\varphi}$ causes a shear rate $\dot{\gamma}$ in the sample

$$
\dot{\gamma}=\frac{\mathrm{d} \gamma}{\mathrm{d} t}=\frac{\dot{\varphi}}{\tan \alpha}
$$

In a step shear rate test, a constant shear rate $\dot{\gamma}_{0}$ is imposed at time $t=0$ and the resulting shear stress measured (stressing test or stress growth test). Figure 1a shows an example for such measurements in the low shear rate regime. Directly after imposition of the shear rate, the shear stress increases rapidly, but finally reaches a plateau after about $8 \mathrm{~s}$. The time range in which the shear stress is time-dependent is called the transient regime. The plateau regime of the shear stress is denoted as steady flow, i.e., both the shear rate and shear stress are constant over time.

After the sample has reached a steady state, the imposed shear rate is set to zero again and one observes a gradual decrease of the shear stress back to zero, which is denoted as stress relaxation. After complete
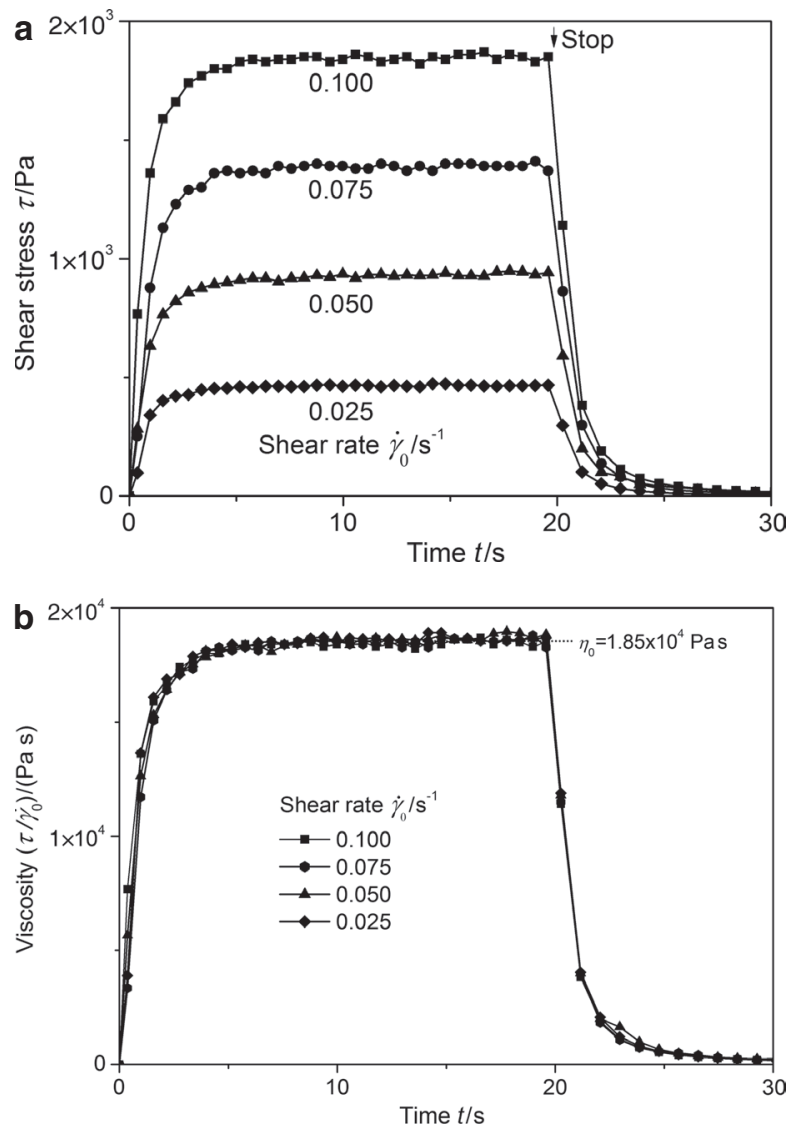

Fig. 1 (a) Shear stress $\tau$ versus time $t$ in step shear rate tests on polyisobutylene Oppanol B10 at $30{ }^{\circ} \mathrm{C}$ and small shear rates. Numbers indicate the constant shear rate $\dot{\gamma}_{0}$ applied at $0 \leq t \leq 20 \mathrm{~s}$. (b) Time-dependent viscosity $\eta$ obtained by normalizing the shear stress $\tau$ from (a) by the constant shear rate $\dot{\gamma}_{0}(0 \leq t \leq 20 \mathrm{~s})$ in the stressing test. All data points collapse onto a single curve, demonstrating that all tests were performed within the linear viscoelastic regime. 
relaxation, a new constant shear rate test may be started, either at the same shear rate to test the reproducibility or at another shear rate to investigate the effect of shear rate (see Fig. 1a).

The shear viscosity $\eta$ is defined as the ratio of shear stress and shear rate

$$
\eta=\frac{\tau}{\dot{\gamma}}
$$

This definition is also used if either one or both vary with time. Then it yields a transient (time-dependent) viscosity. For steady-state flow, the viscosity becomes time-independent and is denoted as steady-state viscosity $\eta_{\mathrm{s}}$. When talking about the shear rate dependence of the viscosity, $\eta(\dot{\gamma})$, one implicitly refers to steady-state values and often omits the suffix s.

In Fig. 1b, the measured shear stress signals are related to the shear rates used in the step shear rate tests. Obviously, all traces collapse onto a single curve. This result is only obtained, however, in the linear viscoelastic regime. Here, the term "linear viscoelastic" means that doubling the shear rate also doubles the magnitude of the transient shear stress, as well as the plateau value (strict proportionality of shear stress and shear rate). As a consequence, the steady-state viscosity is independent of the applied shear rate. The range in which the viscosity is independent of shear rate is also denoted as the Newtonian regime, the viscosity denoted as Newtonian (steady-state) viscosity or as zero-shear (rate) viscosity $\eta_{0}$

$$
\eta_{0}=\lim _{\dot{\gamma} \rightarrow 0} \eta_{\mathrm{s}}(\dot{\gamma})
$$

\subsection{Shear creep test}

Some rheometers allow investigators to impose the shear stress via the applied torque and to measure the resulting shear strain or shear rate. In a step shear stress test, the sample is exposed to a constant shear stress $\tau_{0}$ at $t=0$ (shear creep test). Figure 2a shows an example for the same material as in Fig. 1 . After imposing a shear stress, at small times one also observes a transient regime in which the shear rate [slope of $\gamma(t)]$ decreases with time (hardly to be seen in Fig. 2a because of the large time scale). Finally, a steady state with a constant shear rate is reached. From the latter, the steady-state viscosity is calculated using eq. 4 . Once the imposed torque is removed, a partial reverse shear deformation is observed. The difference between the maximum shear strain before unloading and its final plateau defines the maximum recoverable shear strain
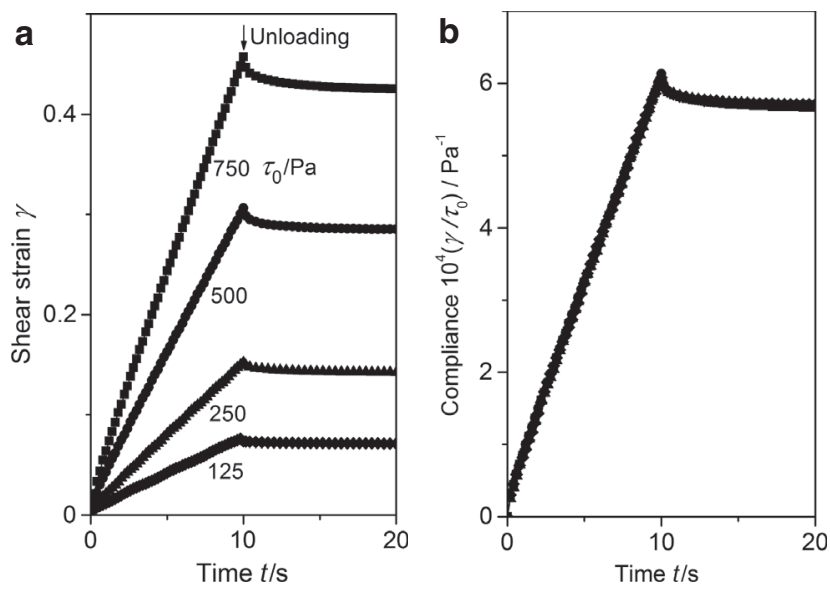

Fig. 2 (a) Shear strain $\gamma$ versus time $t$ in step shear stress tests on polyisobutylene Oppanol B10 at $30{ }^{\circ} \mathrm{C}$. Numbers indicate the constant shear stresses $\tau_{0}$ applied in the interval $0 \leq t \leq 10 \mathrm{~s}$. (b) Shear compliance $/$ obtained by normalizing the shear strain $\gamma$ by the constant shear stresses $\tau_{0}$ in the shear creep tests. All data points collapse onto a single curve, giving evidence that all tests were carried out within the linear viscoelastic regime. 
$\gamma_{r}$. Details of such recovery measurements are outside the scope of this paper, but can be found, e.g., in ref. [23].

The ratio of shear strain and shear stress is called shear compliance $J$ (reciprocal of the shear modulus $G$ ), independently of whether either one or both depend on time

$$
J=\frac{\gamma}{\tau}
$$

In Fig. $2 \mathrm{~b}$ the measured shear strain is divided by the applied constant shear stress during the creep interval. Obviously, all traces collapse onto a single curve. This result again shows that the measurements have been performed in the linear viscoelastic regime. As a consequence, the steady-state viscosity is equal to the zero-shear viscosity $\eta_{0}$. The $\eta_{0}$ values $\left(1.85 \times 10^{4} \mathrm{~Pa}\right.$ s) from Figs. 1 and 2 agree within experimental error. Notably, the shear compliance never becomes time-independent during the creep test. In the steadystate regime, this quantity just increases linearly with time. A stationary value of the compliance is finally observed after unloading the sample.

\subsection{Oscillatory shear measurement}

Strain-controlled rheometers are able to impose a small amplitude oscillatory shear (SAOS) of shear amplitude $\gamma_{0}$ and angular frequency $\omega=2 \pi f\left[\mathrm{rad} \cdot \mathrm{s}^{-1}\right], f\left[\mathrm{~s}^{-1}\right]$ being the frequency of oscillation

$$
\gamma(t)=\gamma_{0} \sin \omega t
$$

In the linear viscoelastic regime, the resulting shear stress oscillates with the same frequency and is proportional to the shear amplitude, but may be phase-shifted. This relation is expressed by the sum of the sine and cosine contributions

$$
\tau(t)=\gamma_{0}\left[G^{\prime} \sin \omega t+G^{\prime \prime} \cos \omega t\right]
$$

with the storage modulus $G^{\prime}$ and the loss modulus $G^{\prime \prime}$. One may rewrite the resulting shear stress as a phaseshifted signal of stress amplitude $\tau_{0}$ and phase angle $\delta$

$$
\tau(t)=\tau_{0} \sin (\omega t+\delta)
$$

The parameters can be calculated from the two moduli

$$
\begin{gathered}
\tan \delta=\frac{G^{\prime \prime}}{G^{\prime}} \\
\tau_{0}=\gamma_{0}\left|G^{*}\right|=\gamma_{0} \sqrt{G^{\prime 2}+G^{\prime \prime 2}}
\end{gathered}
$$

$\left|G^{*}\right|$ is the magnitude of the complex modulus $G^{*}=G+i G^{\prime \prime}$ where $i$ is the imaginary unit [1]. Note that $G^{\prime}, G^{\prime \prime}$, and hence $\delta$ and $\left|G^{*}\right|$ depend on $\omega$. The complex modulus $G^{*}$ and the phase angle $\delta$ can be visualized in a Gaussian diagram with the phase angle $\delta$ given by the angle between the vector describing the complex modulus and the real axis.

If a sinusoidal shear stress of amplitude $\tau_{0}$ and angular frequency $\omega$ is imposed on a sample in a stresscontrolled rheometer, the response is an oscillatory shear strain of amplitude $\gamma_{0}$, phase-shifted by an angle $-\delta$, the moduli $G^{\prime}$ and $G^{\prime \prime}$ remaining the same. The resulting material quantities are the real part $J^{\prime}=G^{\prime} /\left|G^{*}\right|^{2}$ and the imaginary part $J^{\prime \prime}=G^{\prime \prime} /\left|G^{*}\right|^{2}$ of the complex compliance $J^{*}=J^{\prime}+i J^{\prime \prime}$.

Figure 3 shows an example of the oscillating shear stress and shear strain versus time for a viscoelastic melt. Here, the shear strain was imposed and the resulting shear stress was calculated. Note that the diagram 


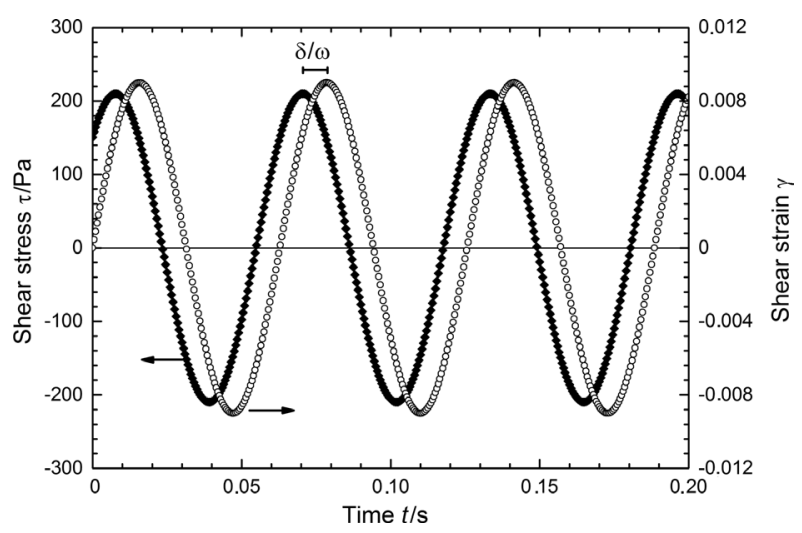

Fig. 3 Example for the sinusoidal stress and strain function of a viscoelastic melt in small-amplitude oscillatory shear (calculated data based on eqs. 7 and 8). The angular frequency is $\omega=100 \mathrm{rad} \cdot \mathrm{s}^{-1}$.

does not show the initial part of the experiment, where the sinusoidal shear is applied for the first time (transient regime), but only a time window in which the response of the material is stationary, i.e., successive cycles show identical behavior. The phase shift between the two signals is clearly seen. The shear stress signal versus time is shifted compared to the shear strain to smaller times by $\Delta t=\delta / \omega$ according to the phase angle $\delta$.

The linear viscoelastic regime for a given sample may be checked experimentally by means of an amplitude sweep of $G^{\prime}$ and $G^{\prime \prime}$ at constant frequency (Fig. 4). Both moduli are amplitude-independent at small strains or stresses, respectively. The limit of linear viscoelasticity shows up as a decrease of the moduli with increasing amplitude. Typically, the limit is first observed for $G^{\prime}$. For the material under investigation, a shear amplitude of $\gamma_{0}=0.5$ is suitable. When approaching the Newtonian regime at low frequencies, the shear amplitude may be increased without surpassing the linear viscoelastic limit. The permitted stress amplitude depends on the absolute value of the complex modulus, see eq. 11. If a constant stress amplitude is imposed, the shear amplitude will increase with decreasing frequency, since $\left|G^{*}(\omega)\right|$ yields smaller values.

\subsection{Empirical Cox-Merz rule}

Based on measurements of the dynamic moduli as a function of angular frequency, one may calculate the frequency dependence of the absolute value of the complex viscosity $\left|\eta{ }^{*}\right|$

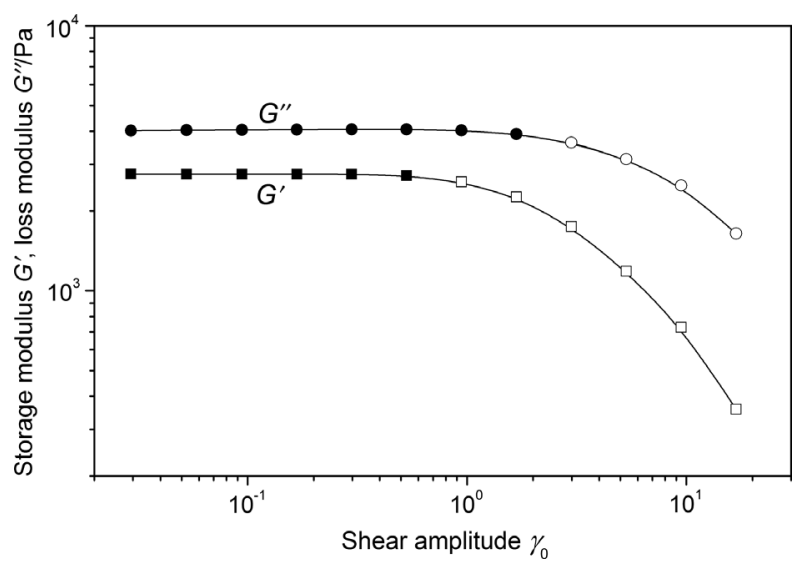

Fig. 4 Storage modulus $G^{\prime}$ and loss modulus $G^{\prime \prime}$ versus shear amplitude $\gamma_{0}$ in an amplitude sweep on an LDPE melt at $150{ }^{\circ} \mathrm{C}$ and $\omega=0.3 \mathrm{rad} \cdot \mathrm{s}^{-1}$ (logarithmic scales). Values deviating more than $5 \%$ from the small amplitude plateaus are indicated by unfilled symbols. Measurements using a shear amplitude of $\gamma_{0} \leq 0.6$ are within the linear viscoelastic regime. 


$$
\left|\eta^{*}(\omega)\right| \equiv \frac{\left|G^{*}(\omega)\right|}{\omega}=\frac{1}{\omega} \sqrt{G^{\prime 2}(\omega)+G^{\prime 2}(\omega)}
$$

Empirically, Cox and Merz [24] found for homogeneous polymer melts an approximate agreement between $\left|\eta^{*}(\omega)\right|$ and the steady-state shear viscosity $\eta_{\mathrm{s}}(\dot{\gamma})$ if the angular frequency is set equal to the shear rate

$$
\left|\eta^{*}(\omega=\dot{\gamma})\right| \cong \eta_{s}(\dot{\gamma})
$$

This rule, linking linear viscoelastic properties (at small oscillatory deformations) to nonlinear properties at high shear rates, has been widely confirmed for homogeneous isotropic liquids. A theoretical justification is possible only for special constitutive laws, see, e.g., ref. [25]. Figure 5 shows an example for a long-chain, branched, low-density polyethylene (LDPE) melt, which has been thoroughly characterized in previous IUPAC projects [26, 27].

On the other hand, the Cox-Merz rule does not work for multiphase liquids like suspensions [28]. Other authors have shown that the data for homogeneous polymer melts may be influenced by flow instabilities like edge fracture, in particular at high shear rates and angular frequencies (in the rubber-like regime) [29, 30]. However, if used with care, which means restricting it to types of material for which its applicability has been verified, the empirical Cox-Merz rule helps to obtain information on the shear viscosity function, solely based on oscillatory measurements, which are conveniently performed over a wide frequency range with high accuracy on small amounts of sample.

\subsection{Comparison of widely used tool geometries}

Table 1 summarizes for the major tool geometries the calculation of rheological parameters from the angular deflection $\varphi$ [rad], amplitude of angular deflection $\varphi_{0}[\mathrm{rad}]$, angular velocity $\Omega=\dot{\varphi}\left[\mathrm{rad} \cdot \mathrm{s}^{-1}\right.$, torque $M[\mathrm{~N} \mathrm{~m}]$, and torque amplitude $M_{0}[\mathrm{~N} \mathrm{~m}]$. We compare cone-plate geometry (CP: plate radius $R$ [m], cone angle $\alpha$ [rad]) with plate-plate geometry (PP: plate radius $R[\mathrm{~m}]$, plate distance $h[\mathrm{~m}]$ ) and concentric cylinder geometry (CC: inner cylinder radius $R_{\mathrm{i}}[\mathrm{m}]$, outer cylinder radius $R_{\mathrm{o}}[\mathrm{m}]$, height of inner cylinder $L[\mathrm{~m}]$ ).

The CP geometry provides the advantage of a practically uniform shear rate and shear stress within the gap for cone angles smaller than about $5^{\circ}$ [31]. Owing to the truncated cone, setting the correct distance between the cone and the plate remains somewhat more demanding than for the plate-plate geometry, for example.

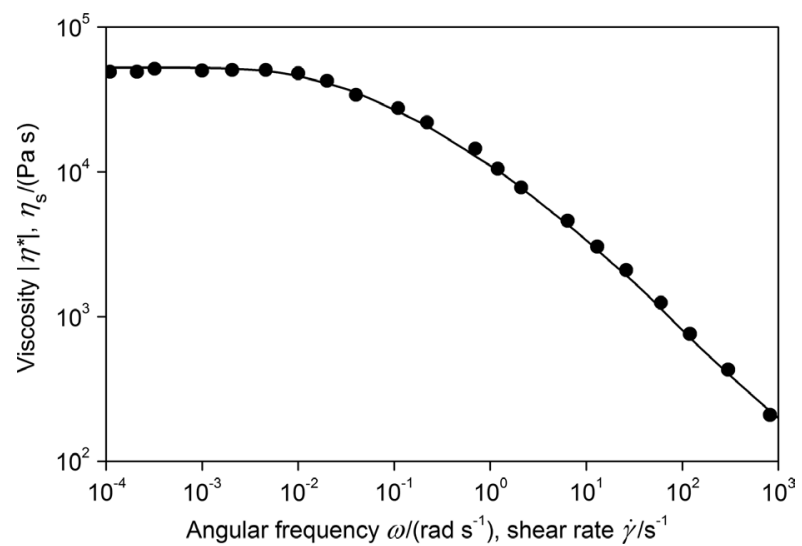

Fig. 5 Comparison of steady shear viscosity $\eta_{\mathrm{s}}$ versus shear rate $\dot{\gamma}$ (circles) [26] and absolute value of the complex viscosity $\left|\eta^{\star}\right|$ versus angular frequency $\omega$ (full line) [27] for an LDPE melt at $150^{\circ} \mathrm{C}$. Both viscosities are compared at $\omega=\dot{\gamma}$, thus demonstrating the validity of the Cox-Merz rule. Details of the measurements are given in the references. 
Table 1 Evaluation of rheological quantities for various tool geometries.

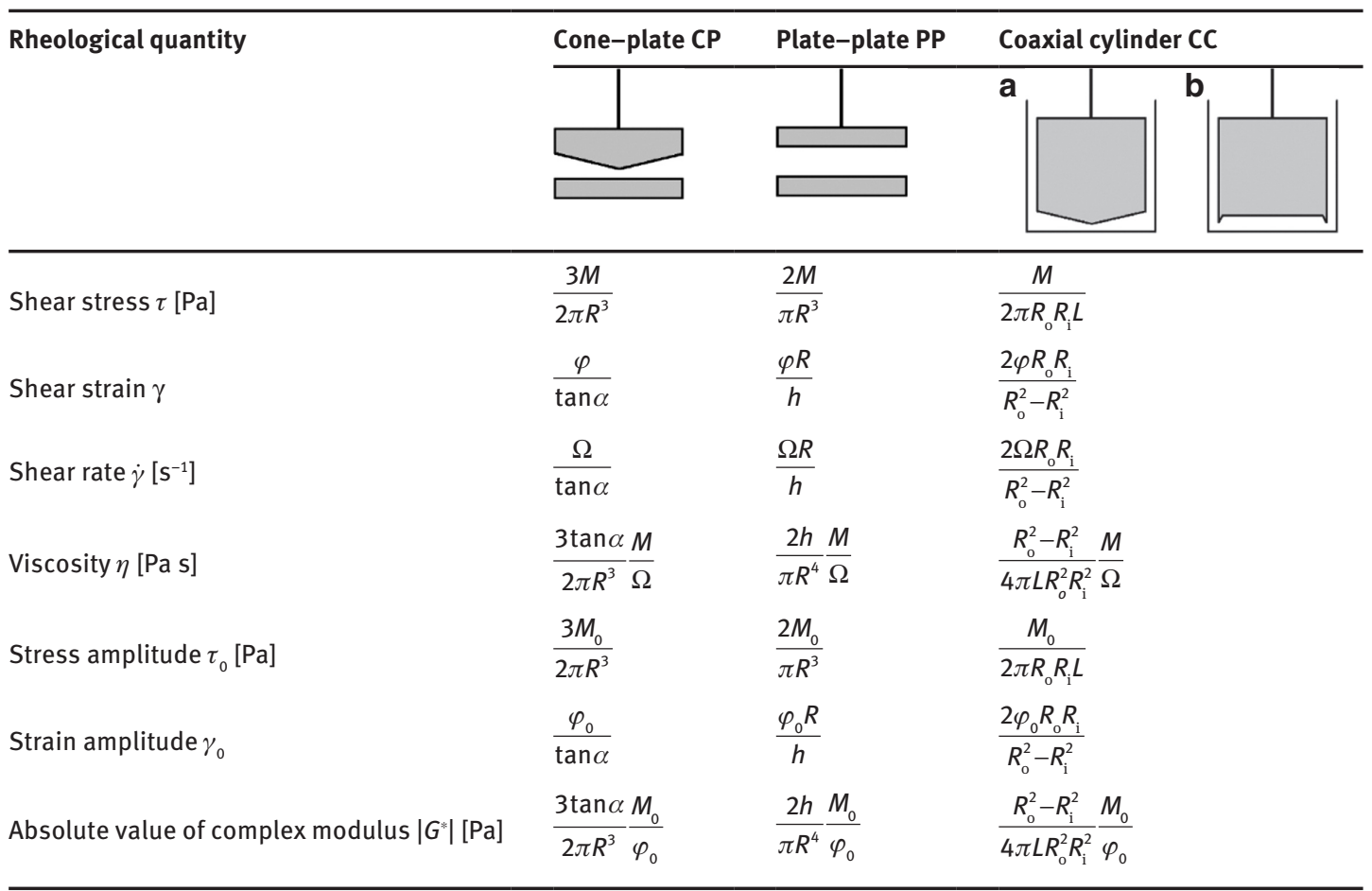

The first four lines refer to steady shear, the last three lines to oscillatory measurements.

Note: Steady shear equations for PP and concentric cylinder geometries assume Newtonian behaviour of the sample and require corrections in the non-Newtonian case (see text). The formulas for shear stress and shear strain for PP and concentric geometry correspond to the values at the rim of the sample.

The PP geometry has the advantage of offering more flexibility in adjusting the gap to compensate for thermal expansion of the sample, or varying the accessible shear rate range, simply by altering $h$. The major drawback of PP tools is that the shear strain increases linearly from zero in the center to a maximum value at the rim. Therefore, one uses the rim shear rate and shear stress as the references and assumes Newtonian behaviour for the shear stress, thus determining an apparent viscosity [1]. In fact, the equations given in Table 1 are valid for the rim, the shear stress and the viscosity being apparent values. Practically, one first determines the apparent viscosity $\eta_{\text {app }}$ (assuming Newtonian behavior) and the apparent shear stress $\tau_{\text {app }}$ as functions of the imposed rim shear rate. In a second step, the assumption is checked and eventually a correction is applied, yielding the true viscosity $\eta_{\mathrm{s}}[2,32]$

$$
\eta_{\mathrm{s}}(\dot{\gamma})=\eta_{\mathrm{app}}(\dot{\gamma})\left[1+\frac{1}{4} \frac{d \log \eta_{\mathrm{app}}(\dot{\gamma})}{d \log \dot{\gamma}}\right]
$$

The correction has to be applied for each (rim) shear rate. If the material is really Newtonian, so that $\eta_{\text {app }}$ is independent of $\dot{\gamma}$, we obtain $\eta_{\mathrm{s}}=\eta_{\text {app }}$.

Two types of coaxial cylinder rheometers have to be distinguished, depending on which part rotates and which is stationary: In the Couette type, the outer cylinder (cup) rotates, whereas for the Searle type the inner cylinder (bob) rotates. In both cases the torque is measured at the bob. The equations given in Table 1 refer to the concentric gap between bob and cup. In reality, there is an additional torque contribution resulting from the bottom of the bob which needs to be taken into account. For example, according to DIN 53019 the cone angle and the gap for geometry (a) may be chosen such that the shear rates in the bottom and in the side gap are identical. Thus, the end effect may be taken into account by using an effective cylinder length $L_{\text {eff }}$ The given equations hold for small gaps $\left(R_{\mathrm{i}}>0.99 R_{\mathrm{o}}\right)$ and assume Newtonian behaviour, since the shear stress in the gap decreases from the cup to the bob wall. The case of non-Newtonian fluids is addressed, e.g., in ref. [2]. 
In a Mooney cell (b), air remains at the bottom of the tool. Therefore, the additional shear stress is strongly reduced and may eventually be neglected.

For all tools, errors in torque and angular deflection measurement as well as in determining the true sample geometry influence the resulting viscosity calculations. For plate-plate (PP) and CP geometries, an error in the true sample radius $R$ in the gap has a strong effect, because the calculated viscosity is proportional to $R^{-4}$ and $R^{-3}$, respectively. For example, if the sample volume between the plates in a PP rheometer is filled only up to $90 \%$ of the plate radius, the obtained viscosity value is too small by $34 \%$ ! Since a large number of parameters influence the viscosity measurement, the typical experimental error of rotational rheometers lies roughly in a range of (1-10) \% [16], depending on the type of material.

\section{Reference materials}

\subsection{Sources of viscosity reference materials}

A convenient method for checking the performance of a rheometer is to load it with a substance of known properties, a so-called reference material, and to compare the measured values with the certified material data. A viscosity reference material should be chemically and physically stable at both the storage and measurement temperatures and under the relevant environmental conditions. It should not be moisture-sensitive (to avoid the plasticizing effect of water). Ideally, the reference material should be stored at constant temperature and humidity, sealed and shielded from light. Most industrial countries have their own institutes of standards, to mention just a few: the National Institute of Standards and Technology (NIST) in the USA, the Bundesanstalt für Materialforschung und -prüfung (BAM) and the Physikalisch-Technische Bundesanstalt (PTB) in Germany, and the Nederlands Meetinstituut (NMi) in the Netherlands. The European Union also has an Institute for Reference Materials and Measurements (IRMM).

The national institutes of standards offer the rather expensive viscosity standards for sale. Many rheologists working with quality control management systems in industry widely use these standards to calibrate their rheometers. The large number of available standards is due to the need for reference materials covering a wide viscosity range and also different levels of viscoelasticity. Typically, these reference materials have a short guaranteed lifetime (up to 1 year). The reliability of the data is checked by annual comparison measurements.

Some rheometer manufacturers also offer reference samples from a larger stock with documented rheological properties. Customers are instructed by means of handbooks and company courses on how to use these reference samples for calibrating their instruments. In addition, calibration and service contracts are offered.

\subsection{Newtonian and non-Newtonian calibration liquids}

Most Newtonian reference liquids are mixtures of mineral or synthetic oils. Various viscosity levels are attained by choosing appropriate average molar masses of the oils. These samples are relatively inert, i.e., the viscosity is constant over long storage times. If the viscosity of a reference material is very temperature sensitive, this property may be used to detect slight deviations in the true sample temperature from the nominal value indicated by the rheometer, thus providing a temperature calibration.

In addition to temperature, the viscosity of non-Newtonian liquids depends on the shear rate (in steady shear) or frequency (in oscillatory shear). This makes the calibration more complex. Rheometer manufacturers offer calibration services and sometimes pertinent viscoelastic calibration standards. For high-temperature calibration, special grades of viscoelastic polyethylene melts are used. A viscoelastic material called "silly putty" is also used for performance tests at room temperature. Silly putty is a polysiloxane, polymerized 
in the presence of boracic acid and filled with silica. It responds as an elastic solid at high frequencies and as viscous liquid at low frequencies.

Certified non-Newtonian liquids are offered by only a few institutions. NIST offers two viscoelastic reference materials: SRM 2490 and 2491. SRM 2490 consists of polyisobutylene dissolved in 2,6,10,14-tetramethylpentadecane, whereas SRM 2491 is a polydimethylsiloxane. According to NIST, these standards are primarily intended for use in calibration and performance checks of instruments that measure viscosity and first normal stress difference in steady shear. In addition, they are used for test measurements of the storage and loss moduli versus frequency at various temperatures, and also to determine the temperature shift factor by applying the time-temperature superposition principle [1]. The certification is valid for 10 years in the case of SRM 2490, and for 2 years in the case of SRM 2491, provided that the SRM is handled in accordance with the storage instructions given in the certificate. NIST monitors both SRM samples over the period of certification. If the certified properties change before expiration of the certificate, NIST will notify the registered purchasers.

The viscosity range of these non-Newtonian NIST standards is shown in Fig. 6, which shows viscosity functions based on oscillatory measurements. SRM 2491 behaves as a non-Newtonian fluid at angular frequencies (shear rates) higher than $1 \mathrm{~s}^{-1}$, whereas SRM 2490 exhibits non-Newtonian behaviour at much lower frequencies. The measured values for SRM 2490 agree well with the NIST certificate, whereas in the case of SRM 2491 some deviations between measured and NIST data appear. Läuger et al. [33] published viscosity data on SRM 2490 that deviate from the NIST certified values. Their data reveal that the steady shear data are inconsistent with the oscillatory shear results. The newest NIST certificate of analysis quotes the steady shear data as "certified values" and the oscillatory shear data solely as "information values".

\section{Explorations of rheometer measurement limits}

\subsection{Theoretical measurement limits}

Various preconditions are required to obtain reliable viscosity data. The major ones are:

- correct tool geometry and gap setting

- constancy of the gap geometry during shear

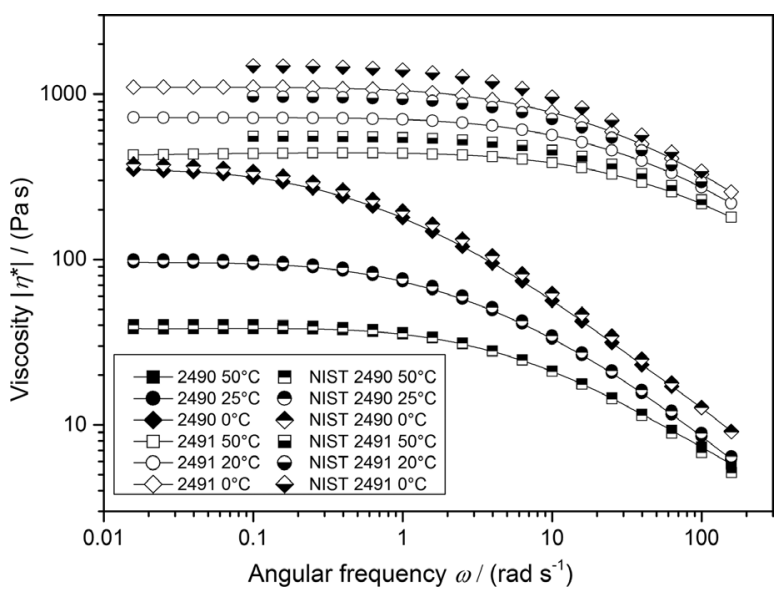

Fig. 6 Magnitudes of the complex viscosity $\left|\eta^{*}\right|$ versus the angular frequency $\omega$ for the two NIST reference materials 2490 (full symbols) and 2491 (open symbols), respectively, each at temperatures $t=0{ }^{\circ} \mathrm{C}$ (diamonds), $25^{\circ} \mathrm{C} / 20^{\circ} \mathrm{C}$ (circles), and $50{ }^{\circ} \mathrm{C}$ (squares). Measurements were made using an Anton Paar MCR 501 rheometer and PP tools $\left(2 R=25 \mathrm{~mm}, h=1 \mathrm{~mm}, \gamma_{0} \leq 0.1\right)$. The semi-filled symbols represent the data of the NIST certificate. 
- correct sample temperature

- stability of sample temperature during shear

The effect of deviations from these preconditions will be addressed later in the text. Based on the design of the rheometer, there is a limited range of operation:

- minimum and maximum angular velocity $\Omega$

- resolution of angular deflection $\varphi$

- $\quad$ minimum and maximum torque $M$

- minimum and maximum angular frequency $\omega$

- maximum allowable normal force $F$

Viscoelastic samples submitted to shear exhibit a normal force in both CP and PP geometry, acting vertically to separate the two parts of the tools [1]. This affects both the bearings of the rotating shaft and the stability of the gap geometry (see Section 5.2). The angular resolution determines the smallest measurable shear strain and also the accuracy of small shear rate measurements (see Section 5.2). The minimum measurable torque is on the one hand governed by the resolution of the torque transducer and on the other hand by possible friction contributions from the bearing.

The minimum and maximum values of angular velocity and torque, $\Omega_{\min }, \Omega_{\max }$ and $M_{\min }, M_{\max }$, respectively, are given in the specifications of the instrument. As an example, we use the specifications for a commercial rheometer of TA Instruments: ARES, Transducer 1, manufactured in $1998, M_{\min }=2 \times 10^{-6} \mathrm{~N} \mathrm{~m}, M_{\max }=$ $2 \times 10^{-2} \mathrm{~N} \mathrm{~m}, \Omega_{\min }=10^{-3} \mathrm{rad} \cdot \mathrm{s}^{-1}, \Omega_{\max }=10^{2} \mathrm{rad} \cdot \mathrm{s}^{-1}$. The theoretical torque range covers 4 powers of magnitude. The range of angular velocities extends over 5 powers of ten. Assuming PP geometry, the range of measurable viscosities based on the equations in Table 1 is given by

$$
\begin{gathered}
\eta_{\text {min }}=\frac{2 h}{\pi R^{4}} \frac{M_{\min }}{\Omega_{\text {max }}} \\
\eta_{\text {max }}=\frac{2 h}{\pi R^{4}} \frac{M_{\text {max }}}{\Omega_{\min }}
\end{gathered}
$$

For a fixed geometry $(h, R)$, the expected measurement range for the viscosity covers 9 powers of 10 . By an adequate variation of the tool geometry, this range may be further broadened. This is demonstrated in Fig. 7, which shows the expected accessible viscosities versus plate radius for two gap heights.

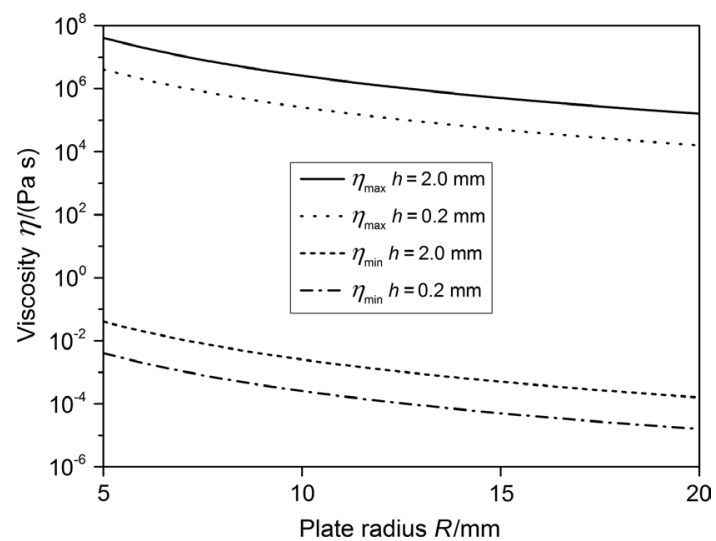

Fig. 7 Theoretical range of possible viscosity measurement versus plate radius $R$ for two gap heights $h=0.2$ and $2 \mathrm{~mm}$, respectively (see eqs. 15 and 16). The underlying instrument specifications are given in the text. 
In reality, the regimes of reliable measurements are distinctly smaller, since side effects come into play. Only a few are mentioned:

- free choice of angular velocity limited to the Newtonian range

- large scatter at the lower torque limit

- low-viscosity samples centrifuged out of the gap at high angular velocities

- dissipative heating of the sample at high angular velocities

- melt fracture at the rim of viscoelastic samples [34]

- occurrence of secondary flows (Taylor vortices) in CC geometry

The problems arising when practical measurement limits are exceeded (in particular, when a large scatter appears at low torques) are demonstrated in Fig. 8. Steady shear measurements on the Newtonian silicone oil Baysilone ${ }^{\circledR}$ M1000 were performed over a broad shear rate range using CP and PP geometry. Whereas a Newtonian viscosity plateau is found in the high shear rate range and the results from the various tool geometries agree within about $\pm 10 \%$, one finds distinct deviations from the Newtonian plateau with decreasing shear rate. Doubling the tool diameter clearly improves the quality of the measurement in the low shear rate regime, i.e., the Newtonian range extends by about one magnitude further into the low shear rate regime. This is due to the strong effect of diameter on the torque for a given shear stress (increase by a factor 8, see Table 1). These measurements show the typical increase of experimental scatter or error when entering the small torque range at low shear rates. A decrease in the measured signal is associated with an increase of the relative errors, thus causing the so-called error trumpet [16]. Since data from the same instrument but from runs using various tools are compared, it is obvious that the low shear rate viscosities are prone to distinct scatter. Thus, a single measurement may be misleading: The PP20 data, for instance, may be misinterpreted as showing an increase of viscosity at small shear rates attaining the slope -1 , typical for a material having a yield stress. In reality, it is an artefact, since the sample being tested is Newtonian.

\subsection{Practical determination of reliable measurement ranges}

In this section we describe procedures used to check empirically the limits of a rotational rheometer. First, we address the viscosity determination in steady shear experiments. If a low viscosity fluid is tested in a strain-controlled instrument, the minimum reliably measurable torque created by the sample is decisive. For a stress-controlled machine, the smallest torque that can be reliably imposed on the sample is important.

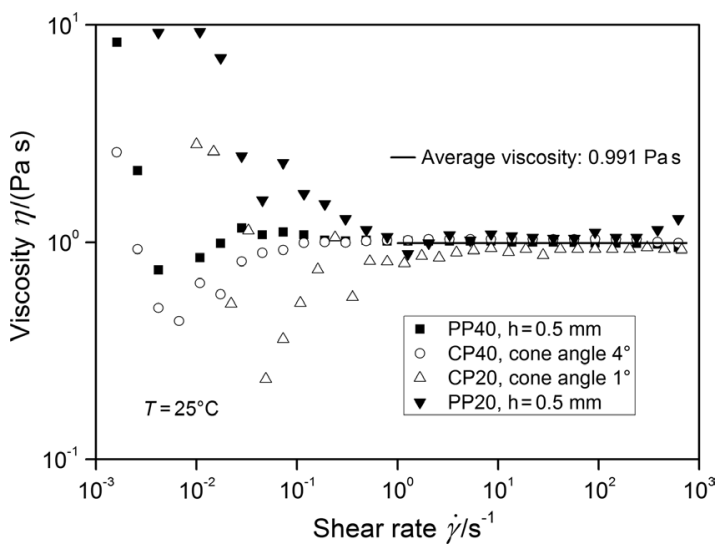

Fig. 8 Viscosity values versus shear rate of a low-viscosity Newtonian oil Baysilone ${ }^{\circledR}$ M1000 as measured using plate-plate (PP20 and PP40) tools (full symbols) of 20 and $40 \mathrm{~mm}$ diameter, respectively (gap height $0.5 \mathrm{~mm}$ ), and using cone-plate (CP20 and CP40) tools (unfilled symbols) of cone angle $1^{\circ}$ and $4^{\circ}$, respectively (plate diameter $20 \mathrm{~mm}$ ). The measurements were performed at room temperature $\left(25^{\circ} \mathrm{C}\right)$ with a Bohlin Gemini 150 rheometer. 
This torque is not identical to the minimum torque of the instrument specification, because friction contributions from the bearing do not necessarily remain constant over time. There is also an upper limit on applicable shear rates, either before the maximum torque is reached or before side effects like sample loss at the rim, melt fracture, and too high normal forces (modifying the gap geometry) come into play. In conclusion, a map exists for each rheometer, tool geometry, and type of material (!), indicating the regime of reliable viscosity measurements (Fig. 9). This map can be explored using reference liquids with known viscosity or viscosity function $\eta(\dot{\gamma})$. Typically, a shear rate (or shear stress) sweep is performed on each of the reference liquids. The resulting viscosity function is compared to the specification of the reference material.

Figures 10 and 11 present examples for the Haake Viscotester 500 (a strain-controlled viscometer mainly for quality control applications). The manual specifies for the angular velocity range: $(0.5-800) \mathrm{rpm}$ $\left[(0.52-84) \mathrm{rad} \cdot \mathrm{s}^{-1}\right]$ and for the torque range: $(0.0001-0.03) \mathrm{N} \mathrm{m}$. Two different $\mathrm{CP}$ systems were used, one with $28 \mathrm{~mm}$ diameter and the cone angle $\alpha=1^{\circ}\left(\mathrm{PK} 1,1^{\circ}\right)$ and another one with $50 \mathrm{~mm}$ diameter and the same cone angle $\left(\mathrm{PK} 5,1^{\circ}\right)$. Based on the velocity and torque ranges, the expected limits for shear rate and shear stress were calculated (Table 2).

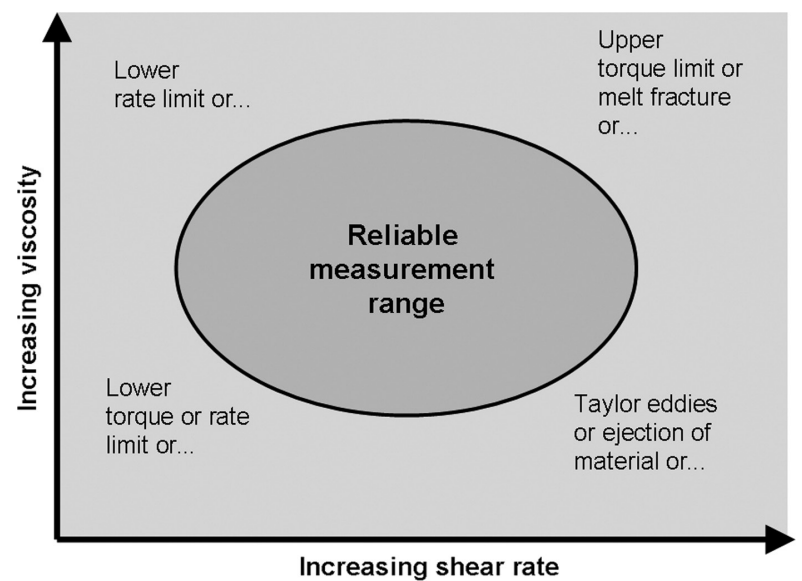

Fig. 9 Schematic of effects that limit the reliable measurement range of a rotational rheometer. There may be additional effects not listed in the scheme.

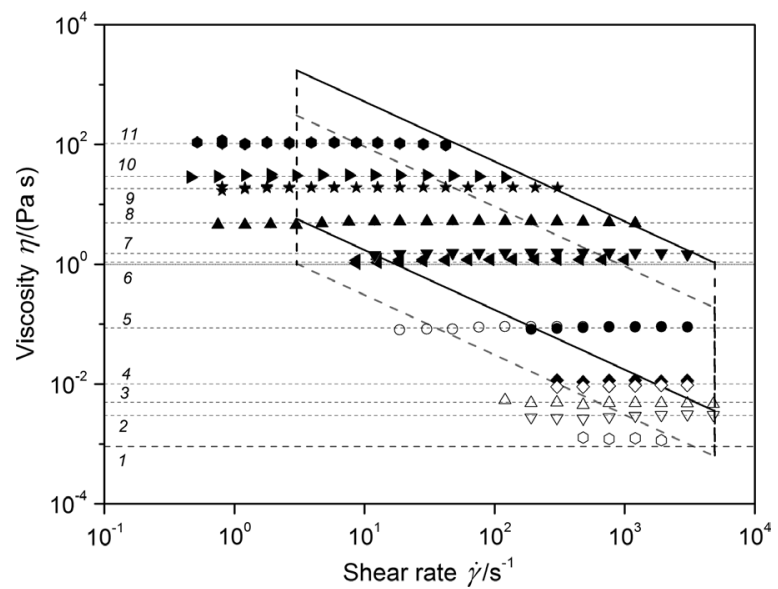

Fig. 10 Viscosity versus shear rate using the Haake Viscotester 500 for the viscosity reference materials listed in Table 3, see the small numbers in italics in the figure. Dotted lines represent the specified viscosity of the standards. Full and broken lines indicate the nominal measurement regimes for CP tools PK1, $1^{\circ}$ and PK5, $1^{\circ}$, respectively (see Table 2 ). Full symbols stem from $\mathrm{PK} 1,1^{\circ}$, and the unfilled symbols represent $\mathrm{PK} 5,1^{\circ}$. 


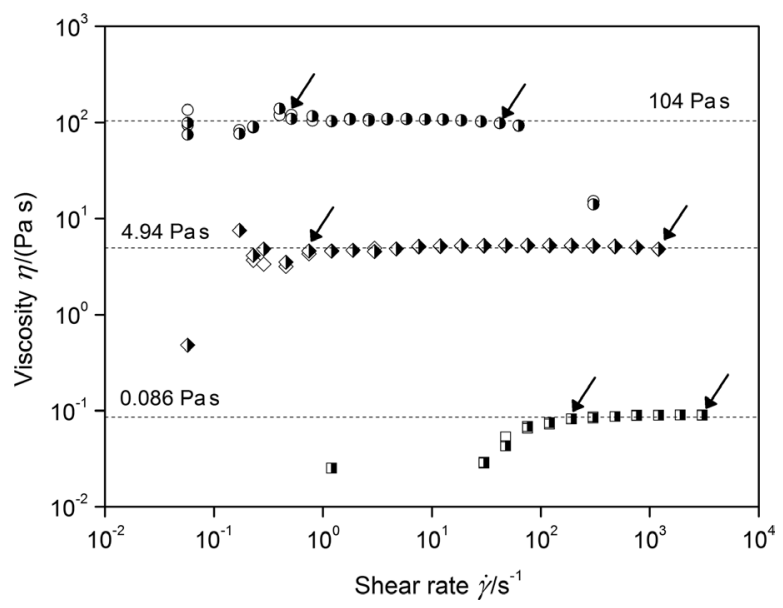

Fig. 11 Viscosity versus shear rate using tool PK1, $1^{\circ}$ for three of the reference materials in more detail (two runs for each sample). For each sample, the arrows indicate the shear rate regime in which the measured viscosity deviates $<10 \%$ from the specified value of the standard (so-called "good data" shown in Fig. 10).

Table 2 Nominal shear rate and shear stress limits of the Haake Viscotester 500 for two different plate diameters and cone angle $1^{\circ}$ based on the angular velocity and torque specifications of the rheometer.

\begin{tabular}{lrr}
\hline & PK1, 10 & PK5, 1 \\
\hline$R /[\mathrm{m}]$ & 0.014 & 0.025 \\
$\dot{\gamma}_{\min } /\left[\mathrm{s}^{-1}\right]$ & 3 & 3 \\
$\dot{\gamma}_{\max } /\left[\mathrm{s}^{-1}\right]$ & 4800 & 4800 \\
$\tau_{\min } /[\mathrm{Pa}]$ & 17.4 & 3.06 \\
$\tau_{\max } /[\mathrm{Pa}]$ & 5220 & 918 \\
\hline
\end{tabular}

Table 3 summarizes the list of Newtonian liquids used to check these theoretical limits. Not all of them are certified viscosity standards. For instance, the Baysilone ${ }^{\circledR}$ oils are not. However, the producer provides a viscosity specification.

In a plot of viscosity versus shear rate (see Fig. 10) these theoretical limits from Table 2 define two boxes with vertical left and right borders (here identical for both geometries because of the constant cone angle) and upper and lower borders of slope -1 in the double logarithmic representation (here different for the two

Table 3 List of Newtonian liquids used to check rheometer limits.

\begin{tabular}{lrrr}
\hline Reference material & No. in Fig. 10 & Specified viscosity/[Pa.s] & Temperature/[ ${ }^{\circ}$ C] \\
\hline Water & 1 & 0.0009 & 25 \\
Baysilone $^{\circledR}$ M3 & 2 & 0.003 & 25 \\
Baysilone $^{\circledR}$ M5 & 3 & 0.005 & 25 \\
Baysilone $^{\circledR}$ M10 & 4 & 0.001 & 25 \\
DKD viscosity standard & 5 & 0.0864 & 20 \\
DKD viscosity standard & 6 & 1.083 & 25 \\
DKD viscosity standard & 7 & 1.504 & 20 \\
Brookfield viscosity Standard & 8 & 4.940 & 25 \\
DKD viscosity Standard & 9 & 18.385 & 20 \\
Brookfield viscosity Standard & 10 & 29.52 & 25 \\
Brookfield viscosity Standard & 11 & 104.0 & 25 \\
\hline
\end{tabular}

Abbreviation DKD stands for Deutscher Kalibrierdienst (Physikalisch-Technische Bundesanstalt (PTB), Braunschweig, Germany). Baysilone $^{\circledR}$ is a trademark of Bayer MaterialScience AG. 
geometries). The tools using the smaller diameter $\left(\mathrm{PK} 1,1^{\circ}\right)$ are represented by the full lines, tools $\mathrm{PK} 5,1^{\circ}$ by broken lines. Only the regimes of "good data" (see Fig. 11) for the various liquids and the two tool geometries are shown in the figure. The specified viscosities of the viscosity reference materials are represented by dotted lines. At least for the PK1, $1^{\circ}$ tool, the upper stress and shear rate limits fit the instrument specification of the manufacturer. Obviously, the rheometer provides reasonable data down to substantially lower shear rates than those indicated in the instrument specification!

Figure 11 shows in more detail the viscosity versus shear rate data for three of the reference materials, measured with geometry PK1, $1^{\circ}$ over a broad shear rate range. For each fluid, a shear rate range can be defined, where the measured viscosity agrees with the specified value of the standard within $\pm 10 \%$ of its specified viscosity (so-called "good data").

Compared to measurements of the steady-state shear viscosity function $\eta_{s}(\dot{\gamma})$, where the control parameter is the shear rate and the viscosity is the target quantity, the situation becomes more complex in small amplitude oscillatory shear experiments. In principle, there are now two control parameters: the angular frequency $\omega$ and the shear amplitude $\gamma_{0}$. The target quantities are the absolute value of the complex modulus $\left|G^{*}(\omega)\right|$, - which, divided by the angular frequency, yields the absolute value of the complex viscosity $\left|\eta^{*}(\omega)\right|$ - and the phase angle $\delta$. Alternatively, the storage and loss moduli $G^{\prime}(\omega)$ and $G^{\prime \prime}(\omega)$ can be evaluated. The limits of the $\left|G^{*}(\omega)\right|$ measurement depend on the torque range and bearing friction of the instrument. Furthermore, it is required that the shear amplitude is chosen such that the measurements are performed in the linear viscoelastic range.

Each rheometer allowing oscillatory measurements can cover only a certain frequency range of operation, $\omega_{\min }$ to $\omega_{\max }$. The upper machine limit $\omega_{\max }$ is governed by the inertia of the moving parts, the maximum torque imposed by the motor, and also the sampling rate and response time of the drive control. The lower machine limit $\omega_{\min }$ depends on the angular resolution and the minimum controllable angular velocity. In reality, there are additional effects that may significantly reduce the frequency range in which reliable data are obtained. As in steady shear experiments, one limitation in the low-frequency range can be that the oscillatory torque is too small. Although oscillatory measurements provide the advantage of detecting the oscillating torque even if there is a high level of noise, there remains a practical limit of resolution. Furthermore, the necessary measurement time may also limit the range of accessible low frequencies. Assuming that the electronics require half a cycle to evaluate amplitude and phase angle of a sine wave, the necessary measuring time for $\omega=0.01 \mathrm{rad} \cdot \mathrm{s}^{-1}$ is calculated to be $t=314 \mathrm{~s}$. Furthermore, a stationary state in the oscillation experiments has to be achieved. For $\omega=0.001 \mathrm{rad} \cdot \mathrm{s}^{-1}$, the measurement time increases to $53 \mathrm{~min}$ ! This effect is on one hand relevant for samples that are not stable over the required time at measurement temperature and environment, and on the other hand one tends to avoid long measurement times in routine testing. Typically, a sweep from high to low frequencies will be used, and the total measurement time is determined by the number of data points per decade and the lowest frequency selected.

The measurement times in the high-frequency regimes are conveniently short, and the torque amplitudes are high. By reducing the shear amplitude, too high torques may be avoided. Principally, this should allow data acquisition with high accuracy. Two effects, however, need to be taken into account. Firstly, if low-viscosity liquids are submitted to high-frequency oscillatory shear, inertia may cause the shear strain in the sample to deviate from the linear vertical profile (the sample "swashing back and forth"). This leads to an apparent increase in the storage modulus $G^{\prime}$ [35]. In a PP configuration, this effect may be reduced by reducing the gap height. Secondly, the torque transducer has a certain angular compliance. The part of the tool attached to the transducer does not remain stationary, but exhibits an angular deflection proportional to the momentary torque. As a consequence, the true shear in the sample is smaller than the nominal one, causing an apparently smaller modulus $\left|G^{*}\right|$. Furthermore, there occurs an error in the measured phase angle. A subsequent correction of the measured data from oscillatory shear is possible, provided the instrument compliance is known [36].

- In summary, the map for reliable $\left|G^{*}\right|$ or $\left|\eta^{*}\right|$ measurements is of larger dimensions than for steady shear viscosity, and a complete exploration becomes rather cumbersome. Some methods are avail- 
able for checking data quality and accessible ranges for reasonable test times: Choice of the optimum tool dimensions: The accessible measurement range is strongly influenced by the tool geometry. To reach higher or lower stress levels in the sample, the tool diameter should be chosen accordingly. When using very small tool dimensions and a tiny sample volume, however, the influence of geometry misalignment and errors in loading becomes more severe. A plate diameter of about $5 \mathrm{~mm}$ appears to be the lower limit for most commercial rheometers and setups, but depending on the alignment, still represents an acceptable compromise for reliable measurements. The diameter can be increased to measure low-viscosity samples. By comparing results from various geometries and measurement modes, one may also estimate the magnitude of errors caused by misalignment, sample loading, etc.

- Improving the signal-to-noise ratio: This can be achieved either by gradually increasing the imposed strain or stress amplitude or by subsequent statistical data analysis. Initially, the imposed amplitudes are chosen to be in a medium range. Then they can be optimized for the specific measurement task. By repeating an experiment several times and averaging the data, it is possible to evaluate and improve noisy data. For example, in oscillatory experiments the number of cycles and data points per cycle can be increased and the applied shear amplitude adjusted on a frequency-dependent basis ("auto-strain technique"). When the latter method is applied, care has to be taken to avoid increasing the amplitude beyond the limit of linear viscoelastic behaviour.

- Time-temperature superposition: This method is often used to determine a material function for a reference temperature $T_{0}$ based on measurements at various temperatures $T$ [8], but over a limited frequency range. The master curve for $T_{0}$ typically provides extended low-frequency data in less time and with higher accuracy than a direct measurement at the reference temperature itself. Furthermore, an inconsistent overlap of the master curve may indicate inaccurate instrument calibration. In principle, only insufficient thermal stability will limit this procedure for fluids in which the microscopic structure does not change with temperature. Some care needs to be taken when testing at various temperatures to avoid errors caused by poor sample temperature equilibration or thermal expansion. The latter effect may be compensated in PP geometry simply by adjusting the gap height via a measurement of the normal force. Some rheometers can do that automatically.

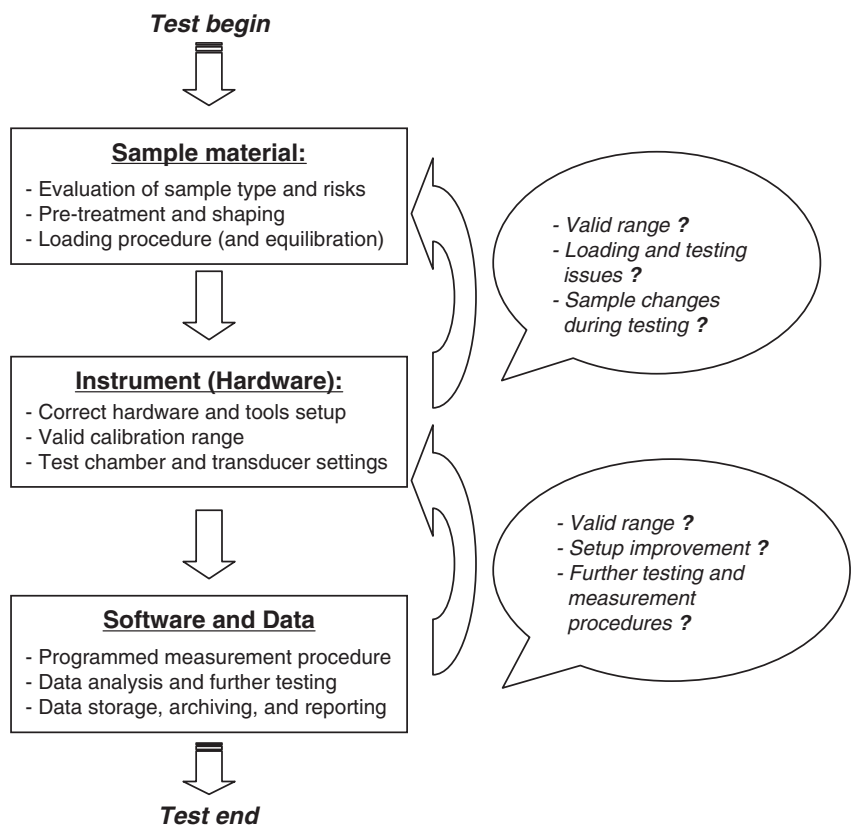

Fig. 12 Flow chart showing a routine test procedure with indication of critical issues. 


\section{Routine calibration procedures}

Good laboratory practice requires routines for monitoring the performance of the rheometer. It is recommended that these routines and their outcome are completely documented, such that unambiguously comparable results will be obtained. A measurement cycle implies optimum handling of the sample, the instrument, and the software (Fig. 12). The tool status needs to be checked for correct geometry, cleanliness, and possible damage (surface and edge). Care is required in mounting the tools to ensure alignment and coaxiality of the two parts, and also to avoid geometrical changes (axial distortion of the shaft) caused by excessive lateral forces. Sample loading and shaping is an issue as well as the appropriate instrument setting. These technical prerequisites are addressed in more detail in the Appendix.

\subsection{Performance check: calibration and verification}

It is mandatory to calibrate the measuring equipment at regular intervals. Since much of the rheometer calibration can be adjusted only by using special tools, it is recommended that a full calibration service (by the manufacturer) is performed at least annually. Normally, an operator cannot perform a calibration himself. The operator therefore needs to monitor the performance regularly, which is called verification. Such verification procedures are typically performed at shorter intervals than full calibration and are less extensive. Verification is normally performed using a reference material (see Section 4). Control of experimental variables such as torque, angular deflection/velocity, temperature, gap setting, frequency, and phase shift is of particular interest.

Verification using a viscosity standard or reference material is performed at a controlled temperature and in a specified environment. Depending on the measurement tools (PP, CP, concentric cylinders), both a suitable reference material and a standardized test procedure should be defined for each rheometer. The test is designed to allow the operator to extract control values that monitor the above-mentioned physical properties of the rheometer. These data are generated at various time intervals and are entered into a control chart (see Fig. 13), which is stored in a database.

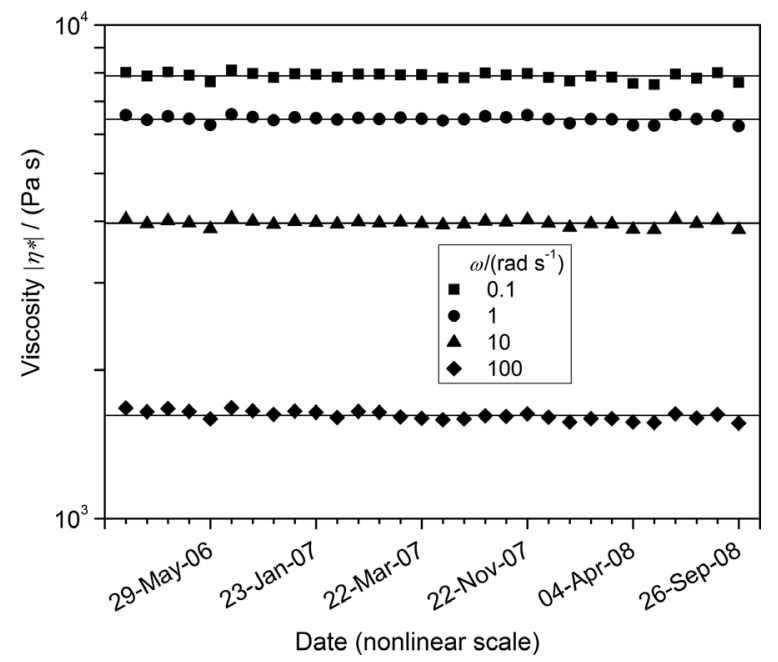

Fig. 13 Control chart of a verification sample (polyethylene at $190^{\circ} \mathrm{C}$ ) showing the measured absolute values of the complex viscosity for angular frequencies $0.1 \mathrm{up}$ to $100 \mathrm{rad} \cdot \mathrm{s}^{-1}$ over a 2 -year period (PP geometry, $2 R=25 \mathrm{~mm}, h=1 \mathrm{~mm}$ ). Note that the time scale is not linear. 
If one of the control values repeatedly lies outside the specified range, the transducer torque, normal force, and torque phase angle have to be calibrated with the help of appropriate weights and/or a calibrated steel bar between the two parts of the tool. Subsequently, the verification test is repeated.

One example of a routine verification test is the small-amplitude oscillatory shear experiment on molten polyethylene depicted in Fig. 13. Here, a frequency sweep was performed in the range of $0.1-100 \mathrm{rad} \cdot \mathrm{s}^{-1}$ at $190{ }^{\circ} \mathrm{C}$ at an imposed shear amplitude of $\gamma_{0}=0.1$ in a nitrogen atmosphere. The plate-plate tool had a diameter of $25 \mathrm{~mm}$, and the gap was $1 \mathrm{~mm}$. The measured absolute value of the complex viscosity $\left|\eta^{*}\right|$ at angular frequencies $\omega=\left(0.1,1.0,10\right.$, and 100) $\mathrm{rad} \cdot \mathrm{s}^{-1}$ represent the control values (alternatively, the absolute value of the complex modulus $\left|G^{*}\right|$ and the phase angle $\delta$ could have been used). The diagram shows these control values plotted against the date of instrument verification.

\subsection{Effect of actual sample temperature on the viscosity}

A quantitative mismatch of viscosities measured by different rheometers or laboratories for identical samples is often due to errors in determining the true sample temperature. For example, a temperature difference of $1 \mathrm{~K}$ may yield a viscosity mismatch between $2 \%$ and $50 \%$, depending on the type of sample and the reference temperature. Figure 14 compares the relative viscosity change $\eta(T) / \eta\left(T_{0}\right)$ as a function of $T-T_{0}[T$ true sample temperature, $T_{0}$ nominal (set) temperature] for various types of polymers. The nominal temperature in Fig. 14 was $190{ }^{\circ} \mathrm{C}$. Most pronounced is the temperature effect for materials investigated near the glass-transition temperature, e.g., see the values of SAN in Fig. 14.

Experimentally, one should distinguish between a temperature mismatch for an isothermal measurement (constant temperature during the whole test) and for a temperature sweep experiment (constant heating/ cooling rate $\mathrm{d} T / \mathrm{d} t$ applied). Possible reasons for errors are

- an offset between set and actual sample temperature,

- radial or axial temperature gradients within the sample [37, 38], or

- a lag of sample temperature behind set heating or cooling rate (only in tests where the temperature is not constant with time)

According to ISO standard $6721-10$ [37], the allowable temperature deviations are: $\pm 0.5^{\circ} \mathrm{C}$ up to $200{ }^{\circ} \mathrm{C}, \pm 1.0^{\circ} \mathrm{C}$ for $(200-300){ }^{\circ} \mathrm{C}$, and $\pm 1.5^{\circ} \mathrm{C}$ for temperatures higher than $300^{\circ} \mathrm{C}$ (measured with a calibrated thermocouple positioned in contact with or embedded in the stationary plate).

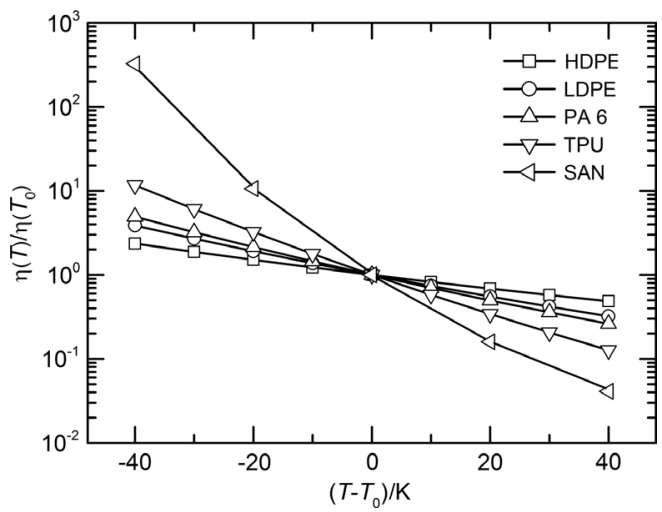

Fig. 14 Relative change of zero-shear viscosity versus temperature difference from reference temperature $t_{0}$ (deviation of temperature from reference temperature). The reference temperature was $190^{\circ} \mathrm{C}$. The materials are high-density polyethylene (HDPE), low-density polyethylene (LDPE), polyamide 6 (PA 6), thermoplastic polyurethane (TPU), and styrene-acrylonitrile copolymer (SAN). 


\subsection{Temperature calibration}

The main reason for a possible difference between set (nominal) temperature and true sample temperature is that the sample temperature cannot be measured within the sample. It is therefore detected by means of a thermo-couple that either contacts the sample holder (tool) or is placed in the oven near the tool. Axial and/or radial gradients of temperature within the sample may be caused by the fact that the shafts of the tools extend outside the oven and are cooled by ambient air, air flow through the air bearing, or simply by contact with the frame of the rheometer. They thus act as heat sinks, the total effect depending on how the shaft is thermally insulated from the tool parts that are in direct contact with the sample. In addition, the efficiency of the oven and whether it uses radiation heating or forced convection by imposed flow of nitrogen plays an important role. Stress-controlled rheometers, which have both the motor drive and the angular deflection detector solely on one side of the tool, allow the stationary plate to be heated electrically or to control its temperature using a Peltier element. The opposite tool part, attached to the motor/transducer combination, cannot be heated directly because it must be able to rotate without any additional torque acting on the shaft except that caused by the sample. Temperature gradients in the sample typically decrease with the flow rate of inert gas (forced convection) and increase at elevated temperatures [38, 39].

An accurate way of checking the true sample temperature is by placing a calibrated thermocouple directly in the (molten) sample or by replacing the polymer sample by, e.g., an aluminum disc, containing one or more properly mounted and calibrated thermocouple(s), see Fig. 15. Using such a setup, a plot of nominal (set) versus actual temperature is obtained (Fig. 16). These data can be used for either correcting the experimental temperature data or for adjusting the necessary set temperatures of the rheometer.

Another way of checking the temperature performance of the instrument is using a temperature calibration sample. For instance, pure indium as test sample shows a sharp transition of the moduli $G^{\prime}$ and $G^{\prime \prime}$ at its melting point of $156^{\circ} \mathrm{C}$ (Fig. 17). At the transition, the specified temperature of the temperature calibration sample allows a direct check of the temperature signal of the rheometer albeit for only one temperature.
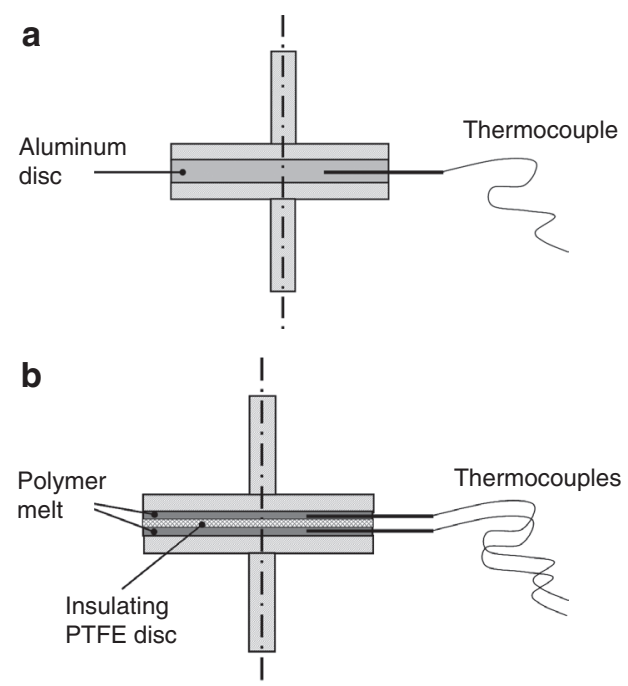

Fig. 15 Measurement setup for (a) the average temperature in the PP gap (aluminum cylinder with thermocouple) and (b) top and bottom temperature (polymer melt discs separated by an insulating poly(tetrafluoroethylene) (PTFE) disc). 


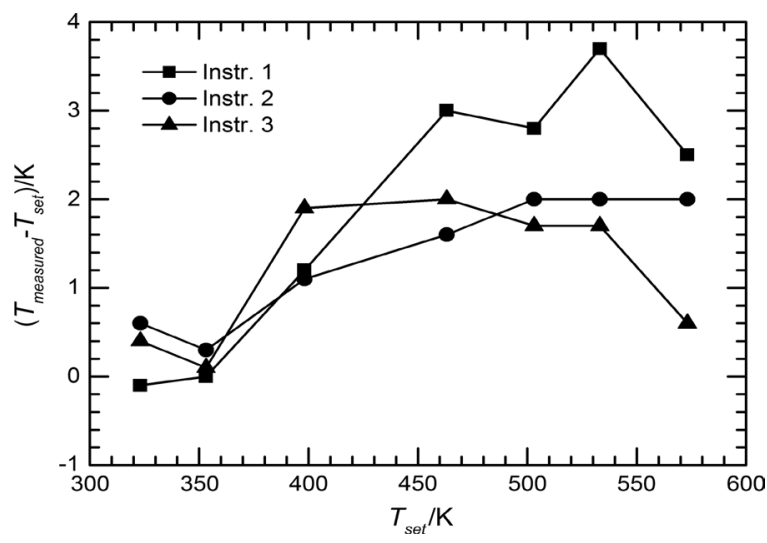

Fig. 16 Difference between set temperature $T_{\text {set }}$ and measured temperature $T_{\text {measured }}$, obtained by using the setup depicted in Fig. 15a. Three different rotational rheometers (ARES, Rheometric Scientific, Inc., Piscataway, NY, USA) were used which are denoted by “Instr. 1", “Instr. 2", and “Instr. 3", respectively. A PP geometry was used.

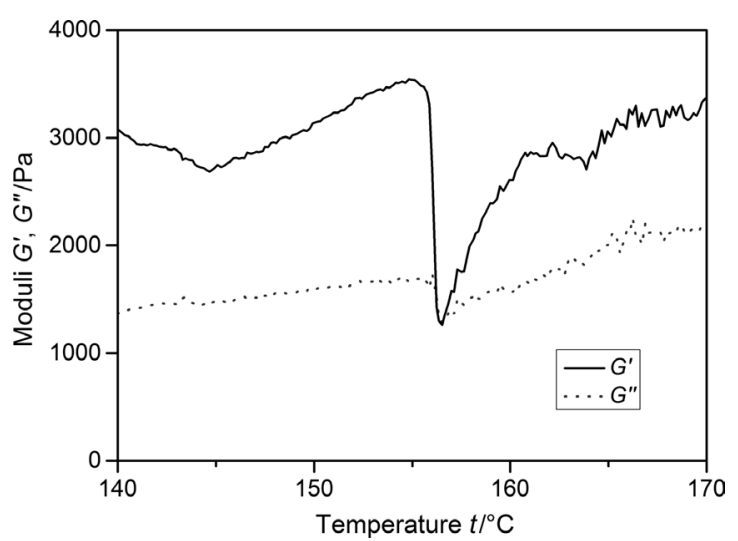

Fig. 17 Storage modulus $G^{\prime}$ and loss modulus $G^{\prime \prime}$ versus temperature $t$ (in ${ }^{\circ} \mathrm{C}$ ) for pure indium measured in small-amplitude oscillatory shear at an angular frequency of $\omega=10 \mathrm{rad} \cdot \mathrm{s}^{-1}$.

\section{Summary}

Various industrial and academic rheology laboratories have merged their expertise in checking and improving the performance of commercial rheometers used for routine and quality control measurements. This paper presents guidelines for a critical assessment of the viscosity data determined by means of such instruments. By using viscosity reference liquids with certified rheological properties, one is able to check the performance of a given rheometer. The general procedure is outlined, including details for sample preparation and loading. It is demonstrated that the regime of reliable measurements, as defined by shear rate range and viscosity level, is not necessarily identical to the specification given by the rheometer manufacturers. This is the main reason why independent techniques that allow an exploration of the reliable measurement regime are important. Viscosity reference materials, predominantly Newtonian oils, are pertinent to check the performance of an instrument. Furthermore, a few viscoelastic samples exist that allow testing the nonNewtonian viscosity behaviour of the sample. In addition, procedures for routinely verifying the accuracy of rheometers used for quality control are addressed. Finally, the role of true sample temperature and its determination by pertinent test settings is described. The true sample temperature may distinctly deviate from the set temperature. Reasons for the deviation are addressed. In summary, the guidelines given in this paper significantly help to improve the accuracy and precision of viscosity measurements in a rotational rheometer. 


\section{Membership of sponsoring body}

Membership of the IUPAC Polymer Division Committee for the period 2014-2015 is as follows:

President: M. Buback (Germany); Vice President: G. Russell (New Zealand); Secretary: M. Hess (Germany); Titular Members: S. Beuermann (Germany); B. Charleux (France); J. He (China/Beijing); R. C. Hiorns (France); G. Moad (Australia); W. Mormann (Germany); M. Sawamoto (Japan); Associate Members: D. Dijkstra (Germany); R. Hutchinson (Canada); I. Lacík (Slovakia); T. Long (USA); D. Smith (USA); J. Vohlídal (Czech Republic); Y. Yagci (Turkey); National Representatives: C.-H. Chan (Malaysia); T. Dingemans (Netherlands); C. dos Santos (Brazil); V. Hoven (Thailand); C.-S. Hsu (China/Taipei); R. Jones (UK); D. S. Lee (Korea); A. Muzafarov (Russia); M. Siddiq (Pakistan).

Acknowledgments: The authors are very grateful to Prof. Clive Bucknall for a critical reading of the manuscript. This multi-laboratory study was performed in the framework of the IUPAC Subcommittee "Structure and Properties of Commercial Polymers" (IUPAC project no. 2007-004-1-400). Various detailed proposals for improving the text by the reviewers are gratefully acknowledged.

\section{Appendix: experimental issues}

The following comments and recommendations summarize experience from an industrial quality control laboratory. It is assumed that the rheometer is correctly set up, including adjustment and alignment of the measurement tools, which need to be free of damage. It is advisable to use standard safety equipment. A major hazard may be the use of large amounts of nitrogen. Thus, adequate ventilation is required, and installation of oxygen depletion monitors is recommended. In addition, harmful solvents may have been used for the preparation of polymer solutions or suspensions. Sometimes, hazardous gases may evaporate during heating of the sample. Care should be taken, and safeguards used in order to avoid touching hot/ cold surfaces, particularly when loading or unloading a sample. In some cases, sample pre-treatment is recommended to shape the sample into a format pertinent to the experiment. This shaping process should not cause changes in molecular structure or phase morphology. Cross-contamination during sample handling must be avoided. The measuring atmosphere (air, nitrogen, etc.) is chosen to provide optimum stability of the sample.

Make sure that the oven is correctly installed to avoid a non-uniform sample temperature. Check the correct placement of the thermocouple and whether it is in good contact with the tool. Some manufacturers allow the operator to choose between oven and tool thermocouples. For melt rheometry, the tool temperature is representative of the sample temperature. The temperature of the oven needs to be in equilibrium, which might take at least $15 \mathrm{~min}$ (preferably $30 \mathrm{~min}$ ) for a PP setup. For CP tools, this time may be in the order of $1 \mathrm{~h}$. After temperature equilibration, the gap for PP or (truncated) CP tools is set to zero. This position is reached when the opposing parts touch and only a small normal force is created. After the mechanical zero point has been defined, the desired gap is set by a relative movement of one part of the tool according to the geometrical requirements. Zeroing the plates is typically done $30 \mathrm{~min}$ after the oven has reached the measurement temperature, either with both plates minimally separated or in contact (if the normal force is automatically adjusted by the instrument). A recent report [18] addresses the effect of speed in zeroing the gap. For high speeds, the flow of residual air/nitrogen out of the gap may cause a measurable normal force before mechanical contact, causing an offset in zero of about $30 \mu \mathrm{m}$. Closing the gap at low speed and choosing a high enough threshold normal force avoids such an offset. Typically, the gap error in PP geometry remains in the order of $1 \%$. For CP tools, a smaller zeroing force is recommended to avoid damage of the truncated cone tip. For the same normal force, the pressure between the touching areas is roughly 100 times higher in CP than in PP geometry, because of the small surface area of the truncated cone.

Each tool requires a specific sample loading procedure. Low-viscosity liquids are injected onto the bottom plate using either a pipette or syringe (with opened gap). Preferably, the amount of material injected is just 
sufficient to fill the final gap. Air bubbles should be avoided. When temperature equilibrium has been established (with the oven closed), the distance between the plates (or between the plate and the cone) is slowly reduced towards the final gap setting. This causes the gap to be filled completely, the sample wetting and covering both adjacent tool surfaces. When additional compression is imposed, the sample will bulge at the rim, causing a viscosity error. The fraction of material outside the gap is proportional to the relative gap reduction. An imposed gap reduction will cause twice as much bulging in a 1-mm nominal gap as in a 2-mm gap. If the gap is not completely filled by the sample, either as a result of using insufficient material or because of an erroneous gap size, the strong radius dependence of the torque comes into play. As a consequence, the measured viscosity in steady shear or the magnitude of the complex viscosity in oscillatory shear will deviate significantly to smaller values. Notably, the phase angle $\delta$ will not be affected.

If the material under investigation is solid at room temperature but molten at elevated measuring temperatures, it may be good practice to produce a sample of correct shape by compression moulding, using the pertinent mould geometry. Another possibility is to produce a sheet of required gap height on a heated press and to cut discs of required diameter from it. Such specimens are ideally suited for PP geometry because they do not contain air bubbles, which are sometimes formed when one loads the rheometer with polymer pellets. Once the sample disc has been inserted onto the lower plate, the oven is closed and the sample is heated up in the rheometer. A flat trim to the plate rim is the preferred sample geometry. If for some reason too much material has been introduced, the oven is opened and the excess material is cut away using a suitable tool. Then the oven is closed again. For CP geometry, it is advisable that the shape of the free surface at the rim should resemble the equatorial surface of a sphere; trimming is thus performed at a gap setting slightly larger than for the measurement. This procedure yields the preferred free surface shape after finally setting the correct CP distance. For Couette and Searle systems, the reader is referred to the instructions of the rheometer manufacturer. A key issue is to avoid air bubbles.

The rheological measurement should be performed within the parameter limits specified by the rheometer manufacturer (see Section 5). In the case of oscillatory shear measurements, it is necessary to know the limit of linear viscoelasticity. Typically, shear amplitude sweeps are carried out to explore the onset of nonlinear behaviour. Such amplitude sweeps should be performed for new types of material at various frequencies to define an appropriate amplitude or range of amplitudes for the measurement of the dynamic moduli versus angular frequency.

Each rheometer manufacturer develops its own user-friendly and modular software. Decisive software parameters that need to be entered correctly into the programme are: temperature, imposed shear amplitude, range of angular frequencies covered, number of frequencies per decade in a logarithmic sweep, and delay time between imposition of a frequency and subsequent data acquisition. Some software allows the oscillation to start and begins to sample data after only half a cycle at the given frequency, then continues for a number of cycles. Some software evaluates the moduli using only part of the cycle. The latter has the advantage of reducing measurement times in the low angular frequency regime, albeit with reduced accuracy. The former can be recommended for higher frequencies, because of the much better signal-to-noise ratio at still acceptable test duration over multiple cycles. It is wise to be aware of what the software is doing. If the software allows modification of the delay and data acquisition parameters, one may use this feature to optimize the quality of the $G^{\prime}$ and $G^{\prime \prime}$ determination. To reduce the measuring time for a given frequency window, there is in principle the possibility of using a multi-frequency mode: the superposition of several frequencies at the same time. The span of the simultaneous frequencies is limited to less than a factor of hundred. In the data file, the instrument software provides not only the parameters and results of the measurement, but normally also the actual gap dimension. This is of relevance if experiments on one sample cover various temperatures (mainly for PP geometry). In this case, the thermal expansion of the sample is detected by the normal force and the gap adjusted accordingly (keeping the normal force zero). The change in actual gap height is automatically taken into account by the software. It should be noted that the results presented in the data files are not necessarily the raw data points measured. Most rheometer software recalculates and averages the raw data to reduce noise and scatter of single data points. These filtered values are displayed in the results spreadsheet. 


\section{References}

[1] C. W. Macosko. Rheology: Principles, Measurements, and Applications, Wiley-VCH Publishers, New York (1994).

[2] M. H. Pahl, W. Gleißle, H. M. Laun. Praktische Rheologie der Kunststoffe und Elastomere, VDI-Verlag, Düsseldorf (1995).

[3] T. G. Mezger. The Rheology Handbook (Coatings Compendia), $2^{\text {nd }}$ ed., Vincentz Network, Hannover (2006).

[4] G. Schramm. A Practical Approach to Rheology and Rheometry, Gebrüder Haake GmbH, Karlsruhe (1994).

[5] R. G. Larson. The Structure and Rheology of Complex Fluids, Oxford University Press, New York (1999).

[6] J. M. Dealy, K. F. Wissbrun. Melt Rheology and Its Role in Plastics Processing, Springer Netherlands, Dordrecht (1999).

[7] J. Mark, K. Ngai, W. Graessley, L. Mandelkern, E. Samulski, J. Koenig, G. Wignall. Physical Properties of Polymers, $3^{\text {rd }}$ ed., Cambridge University Press, Cambridge (UK) (2004).

[8] J. D. Ferry. Viscoelastic Properties of Polymers, $3^{\text {rd }}$ ed., John Wiley, New York (1980).

[9] R. B. Bird, R. C. Armstrong, O. Hassager. Dynamics of Polymeric Liquids, Vol. 1: Fluid Mechanics, Vol. 2: Kinetic Theory, $2^{\text {nd }}$ ed., John Wiley, New York (1987).

[10] R. B. Bird, W. E. Stewart, E. N. Lightfoot. Transport Phenomena, $2^{\text {nd }}$ ed., John Wiley, New York (2007).

[11] M. M. Denn. Process Fluid Mechanics, Prentice-Hall, Englewood Cliffs, NJ (1980).

[12] Z. Tadmor, C. G. Gogos. Principles of Polymer Processing, $2^{\text {nd }}$ ed., John Wiley, Hoboken, NJ (2006).

[13] S. Middleman. Fundamentals of Polymer Processing, McGraw-Hill, New York (1977).

[14] J. M. Dealy. Rheometers for Molten Plastics, Van Nostrand Reinhold, New York (1982).

[15] C. D. Han. Rheology and Processing of Polymeric Materials, Vol. 1: Polymer Rheology, Vol. 2: Polymer Processing, Oxford University Press, New York (2007).

[16] W. Marquardt, J. Nijman. Appl. Rheol. 3(2), 120 (1993).

[17] C. R. Schultheisz, S. D. Leigh. Certification of the Rheological Behavior of SRM 2490, Polyisobutylene Dissolved in 2,6,10,14-Tetramethylpentadecane, NIST Special Publication 260-143 (2002).

[18] G. A. Davies, J. R. Stokes. J. Rheol. 49, 919 (2005).

[19] C. Gabriel, J. Kaschta. Rheol. Acta 37, 358 (1998).

[20] S. S. Velankar, D. Giles. Rheol. Bull. 76 (2), 8 (2007).

[21] S. S. Bafna. Polym. Eng. Sci. 36, 90 (1996).

[22] H. A. Barnes, D. Bell. Korea-Australia Rheol. J. 15, 187 (2003).

[23] C. Gabriel, J. Kaschta, H. Münstedt. Rheol. Acta 37, 7 (1998).

[24] W. P. Cox, E. H. Merz. J. Polym. Sci. 28, 619 (1958).

[25] M. Renardy. J. Non-Newton. Fluid Mech. 68, 133 (1997).

[26] J. Meissner. Pure Appl. Chem. 42, 551 (1975).

[27] H. M. Laun. Rheol. Acta 17, 1 (1978).

[28] W. Gleissle, B. Hochstein. J. Rheol. 47, 897 (2003).

[29] Y. W. Inn, K. F. Wissbrun, M. M. Denn. Macromolecules 38, 9385 (2005).

[30] C. Sui, G. B. McKenna. Rheol. Acta 46, 877 (2007).

[31] D. C. Hylton. Understanding Plastics Testing, Carl Hanser, Munich (2004).

[32] M. T. Shaw, Z. Z. Lui. Appl. Rheol. 16, 70 (2006).

[33] J. Läuger, P. Heyer. Proceedings of the $3^{\text {rd }}$ Brazilian Conference on Rheology, Rio de Janeiro, Brazil, July 12-14, 2006.

[34] K. M. Mattes, R. Vogt, C. Friedrich. Rheol. Acta 47, 929 (2008).

[35] H. M. Laun, R. Hingmann. J. Non-Newton. Fluid Mech. 35, 137 (1990).

[36] S. A. Hutcheson, G. B. McKenna. J. Chem. Phys. 129, 074502:1-14 (2008).

[37] International Organization for Standardization. Plastics-Determination of dynamic mechanical properties, Part 10: Complex shear viscosity using a parallel-plate oscillatory rheometer, ISO 6721-10 (1999).

[38] T. Schweizer. Appl. Rheol. 15, 112 (2005).

[39] D. A. Barker, D. I. Wilson. Rheol. Acta 46, 23 (2006).

Note: Republication or reproduction of this report or its storage and/or dissemination by electronic means is permitted without the need for formal IUPAC or De Gruyter permission on condition that an acknowledgment, with full reference to the source, along with use of the copyright symbol @, the name IUPAC, the name De Gruyter, and the year of publication, are prominently visible. Publication of a translation into another language is subject to the additional condition of prior approval from the relevant IUPAC National Adhering Organization and De Gruyter. 